\title{
Prognostic value of baseline and change in neutrophil-to-lymphocyte ratio for survival in advanced non-small cell lung cancer patients with poor performance status receiving PD-1 inhibitors
}

\author{
Shixue Chen ${ }^{1,2 \#}$, Ruixin Li $^{2 \#}$, Zhibo Zhang ${ }^{3 \#}$, Ziwei Huang ${ }^{2,4}$, Pengfei Cui ${ }^{1,2}$, Wangping Jia ${ }^{1,5}$, \\ Sujie Zhang ${ }^{2}$, Haitao $\mathrm{Tao}^{2}$, Lijie Wang ${ }^{2}$, Xiaoyan $\mathrm{Li}^{2}$, Jinliang Wang ${ }^{2}$, Junxun $\mathrm{Ma}^{2}$, Zhefeng Liu ${ }^{2}$, \\ Di Huang ${ }^{2,4}$, Xuan Zheng ${ }^{1,2}$, Yuichi Saito ${ }^{6}$, Yoshinobu Ichiki ${ }^{7,8}$, Yi $\mathbf{H u}^{2}{ }^{2}$ \\ ${ }^{1}$ Department of Graduate Administration, Chinese PLA General Hospital, Beijing, China; ${ }^{2}$ Department of Medical Oncology, Chinese PLA General \\ Hospital, Beijing, China; ${ }^{3}$ The 78th Group Army Hospital of Chinese PLA, Mudanjiang, China; ${ }^{4}$ School of Medicine, Nankai University, Tianjin, \\ China; ${ }^{5}$ Institute of Geriatrics, Beijing Key Laboratory of Aging and Geriatrics, National Clinical Research Center for Geriatrics Diseases, Second \\ Medical Center of Chinese PLA General Hospital, Beijing, China; ${ }^{6}$ Department of Surgery, Teikyo University School of Medicine, Tokyo, Japan; \\ ${ }^{7}$ Department of General Thoracic Surgery, National Hospital Organization, Saitama Hospital, Wako, Japan; ${ }^{8}$ Second Department of Surgery, \\ University of Occupational and Environmental Health, School of Medicine, Kitakyushu, Japan \\ Contributions: (I) Conception and design: Y Hu, S Chen; (II) Administrative support: Y Hu, J Wang, J Xun, Z Liu; (III) Provision of study materials \\ or patients: S Zhang, H Tao, L Wang, X Li; (IV) Collection and assembly of data: Z Zhang, Z Huang, P Cui, D Huang, X Zheng; (V) Data analysis \\ and interpretation: S Chen, W Jia; (VI) Manuscript writing: All authors; (VII) Final approval of manuscript: All authors. \\ \#These authors contributed equally to this work. \\ Correspondence to: Yi Hu. Department of Medical Oncology, Chinese PLA General Hospital, 28 Fuxing Road, Haidian, Beijing 100853, China. \\ Email: huyi301zlxb@sina.com.
}

Background: Advanced non-small cell lung cancer (NSCLC) patients with poor performance status (PS) are likely to receive programmed cell death 1 (PD-1) inhibitors, despite limited evidence. The aim of the present study was to report the clinical outcomes and potential prognostic biomarkers in advanced NSCLC patients with poor PS receiving PD-1 inhibitors.

Methods: We conducted a retrospective study enrolling 101 advanced NSCLC patients from our hospital. Data of patients with poor PS 2-4 receiving PD-1 inhibitors were retrieved from medical records. Patients were stratified based on dichotomized baseline neutrophil-to-lymphocyte ratio (NLR), change in NLR ( $\triangle \mathrm{NLR} ; 6$ weeks post-treatment NLR minus baseline NLR), and their combination. The receiver-operating characteristic curve was used to assess the best cutoff for NLR. Multivariate Cox analysis was used to evaluate the prognostic value of NLR and $\triangle$ NLR for patients' survival.

Results: The optimal cutoff for NLR was 4.5. The median follow-up was 25.7 months, baseline NLR $\geq 4.5$, and $\triangle N L R \geq 0$, which were independently and significantly associated with shorter overall survival (both $\mathrm{P}=0.002$ ) and progression-free survival ( $\mathrm{P}=0.004$ for $\mathrm{NLR}$ and $\mathrm{P}<0.001$ for $\triangle \mathrm{NLR}$ ). Furthermore, simultaneous elevation of the 2 factors was associated with worsened prognosis; patients with both NLR $\geq 4.5$ and $\triangle N L R \geq 0$ had significantly increased risk of death [hazards ratio (HR): 10.79, 95\% confidence interval (CI): 4.30-27.10] and disease progression (HR: 10.49, 95\% CI: 4.39-25.09), compared with both low NLR and $\triangle$ NLR patients. Patients with either NLR $\geq 4.5$ or $\triangle N L R \geq 0$ showed an intermediate risk for death (HR: 3.12, 95\% CI: 1.35-7.21) and progression (HR: 3.45, 95\% CI: 1.62-7.36).

Conclusions: High baseline NLR and increased post-treatment NLR might aid in the stratification of high progression and death risk groups in advanced NSCLC patients with poor PS receiving PD-1 inhibitors.

^ ORCID: Shixue Chen, 0000-0001-5480-6681; Zhibo Zhang, 0000-0001-8534-7190; Yi Hu, 0000-0001-9319-5692. 


\begin{abstract}
Keywords: Neutrophil-to-lymphocyte ratio (NLR); programmed cell death 1; performance status (PS); lung
\end{abstract} cancer; biomarkers

Submitted Sep 24, 2020. Accepted for publication Mar 02, 2021.

doi: $10.21037 /$ tlcr-21-43

View this article at: http://dx.doi.org/10.21037/tlcr-21-43

\section{Introduction}

Up to $34 \%$ of lung cancer patients have poor Eastern Cooperative Oncology Group performance status (ECOG PS) 2-4 at the time of diagnosis (1). For advanced nonsmall cell lung cancer (NSCLC) patients with poor PS, targeted therapy with low toxicity is recommended for a small number of patients who have detectable genetic mutations. For patients with PS $3-4$, chemotherapy is no longer recommended, and the best supportive care recommended is standard palliative radiotherapy $(1,2)$. The prognosis of this specific population is generally poor in the pre-immunotherapy era.

The success of anti-programmed cell death 1 (PD-1) therapy has revolutionized the treatment landscape for NSCLC patients, however the survival benefit in poor PS patients remains controversial, mainly due to the inclusion criteria of most clinical trials of patients with PS $0-1$. So far, only 4 prospective trials, including a small number of PS 2 patients receiving immunotherapy, have been published, with the overall response rate (ORR), median progressionfree survival (PFS), and overall survival (OS) ranging from $20 \%$ to $27 \%, 3.6$ to 4.4 months, and 4 to 9.8 months, respectively (3-6). Therefore, it is an urgent demand for NSCLC patients with PS $2-4$ to investigate the safety and efficacy of immunotherapy.

A large proportion of patients receiving PD-1 inhibitors do not experience survival benefits due to the substantial heterogeneity of tumor tissue and the immune system. Approved biomarkers, including PD-L1, tumor mutational burden (TMB), and mismatch repair (MMR)/microsatellite instability (MSI), have limited predictive value because of the lack of tumor tissue and molecular analyses (7). Therefore, it is critical to explore non-invasive and costeffective potent biomarkers that could predict treatment outcomes dynamically. And then, some researchers pay attention to neutrophil-to-lymphocyte ratio (NLR), defined as the absolute neutrophil count (ANC) divided by the absolute lymphocyte count. NLR is simple, easy-tocalculate, combining at least 2 blood cell subpopulations, and is accessible from routine complete blood cell counts, which could reflect the systemic inflammatory status and has been proved to be associated with poor outcomes of treatment for NSCLC patients (8-12). So we adopted it as less-invasive and non-expensive biomarker of immunotherapy for NSCLC patients with PS 2-4.

In the present retrospective study, we aimed to investigate the relationship between NLR and clinical outcomes of antiPD-1 therapy for advanced NSCLC patients with poor PS.

We present the following article in accordance with the REMARK reporting checklist (available at http://dx.doi. org/10.21037/tlcr-21-43).

\section{Methods}

\section{Patients}

The Institutional Review Board of the Chinese People's Liberation Army General Hospital (Beijing, China) approved the review of the medical records (approval number: S2018092-01). All procedures performed in this study involving human participants were in accordance with the Declaration of Helsinki (as revised in 2013). Individual consent for this retrospective analysis was waived. A total of 704 consecutive patients with histologically confirmed advanced NSCLC receiving PD-1 inhibitor treatment between January 1, 2015 and December 31, 2019 were reviewed; of these patients, 101 patients with poor ECOG PS 2-4 were included in the final analysis. Demographic, clinical, and pathological data were also collected. Tumor assessment was performed at baseline and then after every 2 cycles of anti-PD-1 treatment, which was generally every 6 weeks. Radiographic responses were classified according to the response evaluation criteria in solid tumors (RECIST 1.1).

\section{Data collection}

Complete blood cell counts with differential counts at baseline (within 7 days before the first anti-PD-1 treatment) and 6 weeks after the first anti-PD-1 treatment were 
extracted. We counted the number of neutrophil and lymphocyte in serum, and calculated the ratio of neutrophil/ lymphocyte. Baseline NLR was defined as the ratio prior anti-PD-1 treatment, furthermore $\triangle \mathrm{NLR}$ was defined as 6 weeks post-treatment NLR minus baseline NLR.

To further evaluate the prognostic relevance of a combination of baseline NLR and $\triangle$ NLR, patients were stratified based on baseline NLR $\geq 4.5$ and $\triangle N L R \geq 0$, characterizing 3 groups (group a, zero factors; group b, 1 factor; group c, 2 factors). Patients with NLR $<4.5$ and $\triangle N L R<0$ were included in group a; either NLR $\geq 4.5$ or $\triangle \mathrm{NLR} \geq 0$ were included in group b; NLR $\geq 4.5$ and $\Delta \mathrm{NLR}$ $\geq 0$ were included in group c.

\section{Statistical analysis}

OS was calculated from the first PD-1 inhibitor treatment until death or the last follow-up (censored). PFS was derived from the first PD-1 inhibitor treatment until the first documented disease progression (PD), death, or the last follow-up (censored). The ORR was the proportion of patients with best responses of complete response (CR) or partial response (PR); the disease control rate (DCR) was the proportion of patients with $\mathrm{CR}, \mathrm{PR}$, or stable disease.

Patients' clinical characteristics were compared using Fisher's exact test for discrete variables and the Wilcoxon rank sum test for continuous variables. The optimal cutoff value for baseline NLR was evaluated by the analysis of the time-dependent receiver-operating characteristic (ROC) curve, and the area under the curve (AUC) was determined. Survival analyses were performed using the Kaplan-Meier method and the log-rank test. Variables with $\mathrm{P}<0.05$ in the univariate analysis were included in the final multivariate Cox model. All $\mathrm{P}$ values were from 2-sided tests and were considered statistically significant at $\mathrm{P}<0.05$. SPSS version 25.0 with Stata version 15.1, was used for the statistical analyses.

\section{Results}

\section{Patient characteristics}

Between January 1, 2015 and December 31, 2019, 704 advanced NSCLC patients received PD-1 inhibitors at the Chinese People's Liberation Army General Hospital. Of these, 101 patients with poor ECOG PS 2-4 were included in the study. The patients' demographic and clinical characteristics are summarized in Table 1 . The majority of the 101 patients had an ECOG PS score of 2 (65.3\%), followed by scores of $3(30.7 \%)$, and 4 (4.0\%). Forty-nine patients were $\geq 65$ years of age (48.5\%), 72 were male $(71.3 \%), 29$ were female $(28.7 \%), 67$ were diagnosed with adenocarcinoma $(66.3 \%)$, and 90 had stage IV disease (89.1\%). Twenty-seven (26.7\%), 32 (31.7\%) and 42 (41.6\%) patients received immunotherapy as systemic first, second, third and subsequent line therapy, respectively. Forty-nine (48.5\%) patients received nivolumab and 47 (46.5\%) received pembrolizumab. The PD-1 inhibitor agents (others) were three PD-1 inhibitors produced by Chinese pharmaceutical companies, i.e., sintilimab (1 patients), camrelizumab (1 patients), toripalimab (3 patients). As of July 20, 2020, 13 $(12.9 \%)$ patients were still alive. The median follow-up was 25.7 months [95\% confidence interval (CI): 13.4-37.9], the median OS and PFS were 9.0 (95\% CI: 6.3-11.8) and 4.3 (95\% CI: 3.2-5.3) months, respectively. The ORR and DCR were $19 \%$ and $63 \%$, respectively (Table 2).

Our univariate analysis showed that only high levels of baseline NLR and increased post-treatment NLR were significantly associated with OS [hazards ratio (HR): 2.28 , 95\% CI: $1.41-3.67, \mathrm{P}<0.001$ for NLR; HR: $2.11,95 \%$ CI: 1.31-3.38, $\mathrm{P}=0.002$ for $\triangle \mathrm{NLR}$ ) and PFS (HR: 2.21, $95 \%$ CI: $1.41-3.48, \mathrm{P}<0.001$ for NLR; HR: $1.94,95 \%$ CI: $1.25-$ 3.01, $\mathrm{P}=0.003$ for $\triangle \mathrm{NLR}$ ) (Table $\mathrm{S} 1$ ).

\section{Baseline NLR and survival}

The time-dependent ROC curve was used to determine the optimal cutoff for survival (Figure S1). The AUC was $0.711(\mathrm{P}=0.050)$. The optimal cutoff of baseline NLR, as determined by the AUC, was 4.5 . Forty-five $(44.6 \%)$ and 56 patients $(55.4 \%)$ presented with baseline NLR $<4.5$ and $\geq 4.5$, respectively. There were no significant differences in baseline characteristics between the low and high NLR groups.

Median OS and PFS were 12.9 (95\% CI: 9.616.3 months) and 6.6 months (95\% CI: 4.4-8.8 months), respectively, for patients with NLR $<4.5$, and $6.1(95 \%$ CI: 4.8-7.3 months) and 2.9 months (95\% CI: $2.4-$ 3.4 months), respectively, for patients with NLR $\geq 4.5$ (both $\mathrm{P}<0.001$ ) (Figure $1 A, B$ ). The multivariate analysis showed that only high levels of NLR were significantly associated with increased risk of death (HR: 2.32, 95\% CI: 1.38-3.91, $\mathrm{P}=0.002$ ) and disease progression (HR: 2.01, 95\% CI: 1.24 $3.24, \mathrm{P}=0.004)$ (Table 3).

The ORR (29\% vs. $11 \%, \mathrm{P}=0.039)$ and DCR (78\% vs. $52 \% ; \mathrm{P}=0.020)$ were also significantly higher in the low 
Table 1 Baseline patient demographic and clinical characteristics

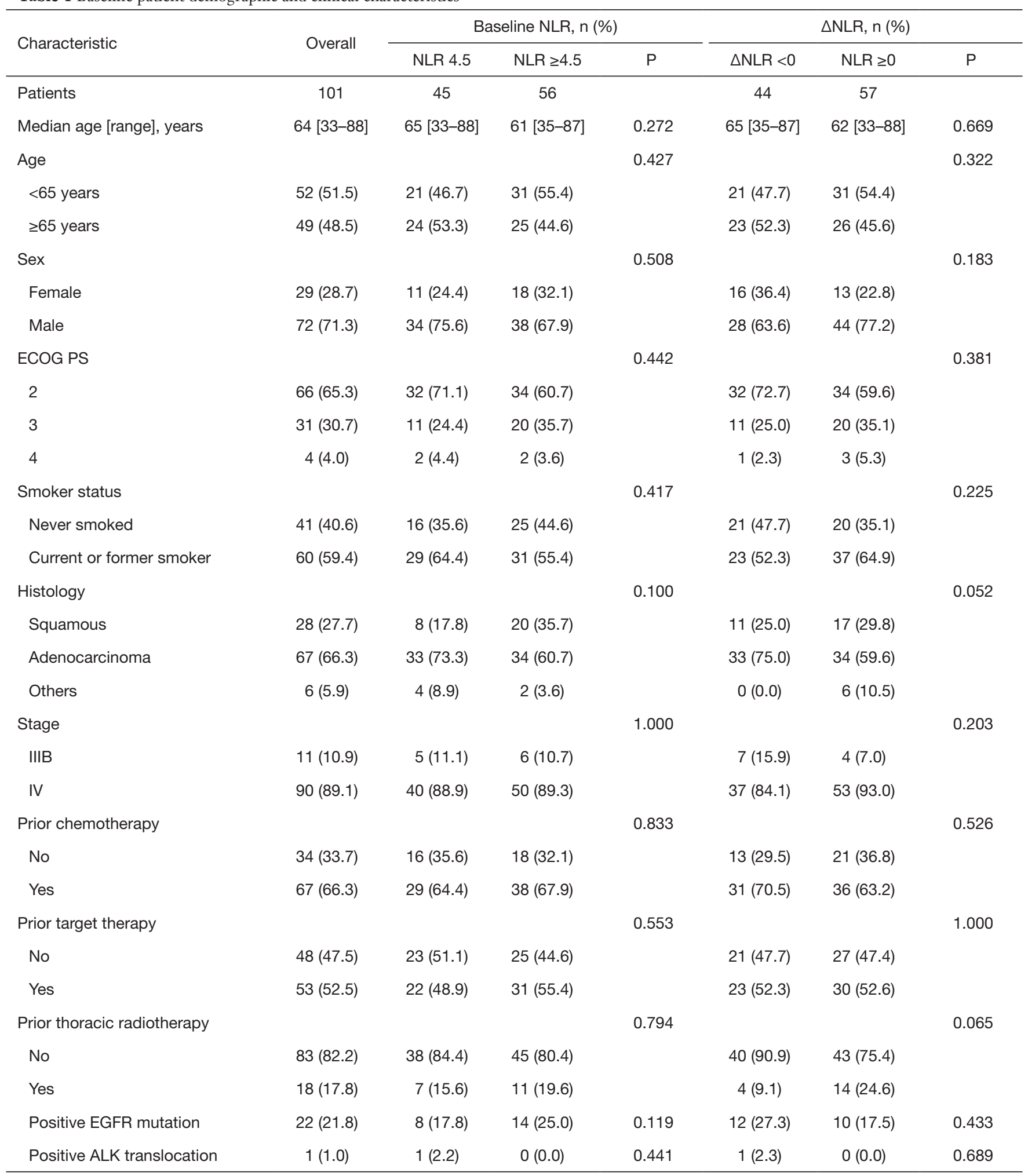

Table 1 (continued) 
Table 1 (continued)

\begin{tabular}{|c|c|c|c|c|c|c|c|}
\hline Characteristic & Overall & \multicolumn{3}{|c|}{ Baseline NLR, n (\%) } & \multicolumn{3}{|c|}{$\triangle \mathrm{NLR}, \mathrm{n}(\%)$} \\
\hline PD-L1 expression & & & & 0.463 & & & 0.241 \\
\hline$<1$ & $15(14.9)$ & 7 (15.6) & $8(14.3)$ & & $5(11.4)$ & $10(17.5)$ & \\
\hline $1-49$ & $17(16.8)$ & $7(15.6)$ & $10(17.9)$ & & $6(13.6)$ & $11(19.3)$ & \\
\hline Not examined & $54(53.5)$ & $27(60.0)$ & $27(48.2)$ & & $23(52.3)$ & $31(54.4)$ & \\
\hline Combined with other therapy & & & & 0.841 & & & 0.688 \\
\hline No & $47(46.5)$ & $20(44.4)$ & $27(48.2)$ & & $19(43.2)$ & $28(49.1)$ & \\
\hline Yes & $54(53.5)$ & $25(55.6)$ & $29(51.8)$ & & $25(56.8)$ & $29(50.9)$ & \\
\hline Yes & $37(36.6)$ & $13(28.9)$ & $24(42.9)$ & & $15(34.1)$ & $22(38.6)$ & \\
\hline Immunotherapy as systemic & & & & 0.158 & & & 0.670 \\
\hline 1 & $27(26.7)$ & $14(31.1)$ & $13(23.2)$ & & $11(25.0)$ & $16(28.1)$ & \\
\hline 2 & $32(31.7)$ & $17(37.8)$ & $15(26.8)$ & & $16(36.4)$ & $16(28.1)$ & \\
\hline$\geq 3$ & $42(41.6)$ & $14(31.1)$ & $28(50.0)$ & & $17(38.6)$ & $25(43.9)$ & \\
\hline PD-1 inhibition agent & & & & 0.293 & & & 0.841 \\
\hline Nivolumab & $49(48.5)$ & $20(44.4)$ & $29(51.8)$ & & $23(52.3)$ & $26(45.6)$ & \\
\hline Pembrolizumab & $47(46.5)$ & $21(46.7)$ & $26(46.4)$ & & $19(43.2)$ & $28(49.1)$ & \\
\hline
\end{tabular}

ALK, anaplastic lymphoma kinase; CNS, central nervous system; ECOG PS, Eastern Cooperative Oncology Group Performance Status; EGFR, epidermal growth factor receptor; NLR, neutrophil-to-lymphocyte ratio; PD-1, programmed cell death 1; $\triangle N L R, 6$ weeks post-treatment NLR minus baseline NLR.

NLR group compared with the high NLR group (Table 2).

\section{$\triangle N L R$ and survival}

Previous studies have suggested that the elevation of posttreatment NLR, compared with pretreatment NLR, is associated with carcinogenesis and poor prognosis (13-15). Based on this, we categorized patients into decreased $(\triangle \mathrm{NLR}<0)$ and increased $(\Delta \mathrm{NLR} \geq 0)$ post-treatment NLR group. We found that $43.6 \%$ and $56.4 \%$ of patients presented with $\Delta$ NLR $<0$ and $\Delta$ NLR $\geq 0$, respectively. However, there were no statistically significant differences in baseline characteristics between the 2 groups.

The median OS and PFS were 12.2 (95\% CI: 7.916.6 months) and 7.1 months (95\% CI: 4.7-9.5 months), respectively, for patients with $\Delta \mathrm{NLR}<0$, and 6.7 (95\% CI: 5.5-8 months) and 3.3 months (95\% CI: 2.6-4.1 months), respectively, for patients with $\triangle \mathrm{NLR} \geq 0 ; \mathrm{P}=0.002$ for $\mathrm{OS}$ and $\mathrm{P}=0.003$ for PFS) (Figure $1 C, D$ ). The multivariate analysis showed that, prior to chemotherapy, and receiving antibiotics within 1 month before anti-PD-1 initiation (mainly due to infection and inflammation caused by nontumor factors) were significantly associated with worse PFS (Table 4). Notably, post-treatment increased NLR levels was independently associated with increased risk of death (HR: 2.20, 95\% CI: $1.35-3.58, \mathrm{P}=0.002)$ and disease progression (HR: 2.52, 95\% CI: 1.56-4.08, $\mathrm{P}<0.001$ ) (Table 4).

Although the ORR (27\% vs. $12 \%, \mathrm{P}=0.078)$ and DCR $(70 \%$ vs. $58 \%, \mathrm{P}=0.299)$ were higher in the decreased posttreatment NLR $(\triangle \mathrm{NLR}<0)$ group compared with the 
Table 2 Relationship between groups and response to anti-PD-1 treatment

\begin{tabular}{|c|c|c|c|c|c|c|c|c|c|c|c|}
\hline Variable & $\begin{array}{l}\text { Overall } \\
(n=101)\end{array}$ & \multicolumn{3}{|c|}{ Baseline NLR, n [\%] } & \multicolumn{3}{|c|}{$\Delta \mathrm{NLR}, \mathrm{n}[\%]$} & \multicolumn{4}{|c|}{$N L R+\Delta N L R, n[\%]$} \\
\hline $\mathrm{CR}$ & $0[0]$ & $0[0]$ & $0[0]$ & & $0[0]$ & $0[0]$ & & $0[0]$ & $0[0]$ & $0[0]$ & \\
\hline PD & 35 [35] & 10 [22] & 25 [45] & & $13[30]$ & 22 [39] & & $2[13]$ & 19 [32] & 14 [52] & \\
\hline $\mathrm{NA}^{\dagger}$ & 2 [2] & $0[0]$ & $2[4]$ & & $0[0]$ & 2 [4] & & $0[0]$ & $0[0]$ & $2[7]$ & \\
\hline ORR & 19 [19] & 13 [29] & $6[11]$ & $0.039^{\star}$ & $12[27]$ & $7[12]$ & 0.078 & 8 [53] & $9[15]$ & $2[7]$ & $0.002^{*}$ \\
\hline
\end{tabular}

*, $\mathrm{P}<0.05$ indicates statistical significance; ${ }^{\dagger}$, patients who had a baseline assessment by investigator review but no post-baseline assessment by data cutoff date, including discontinuation or death before first post-baseline scan. BOR, best overall response; CR, complete response; DCR, disease control rate; NA, not assessable; NLR, neutrophil-to-lymphocyte ratio; ORR, objective response rate; PD, progressive disease; PR, partial response; SD, stable disease; $\triangle N L R, 6$ weeks post-treatment NLR minus baseline NLR.

increased post-treatment NLR $(\Delta \mathrm{NLR} \geq 0)$ group, there were no statistically significant differences (Table 2).

\section{Baseline NLR and $\triangle N L R$ and survival}

To further evaluate the prognostic relevance of a combination of baseline NLR and $\triangle \mathrm{NLR}$, patients were stratified based on baseline NLR $\geq 4.5$ and $\triangle N L R \geq 0$, characterizing 3 groups (group a, zero factors; group b, 1 factor; group c, 2 factors). Fifteen patients with NLR $<4.5$ and $\triangle$ NLR $<0$ were included in group a; 59 patients with either NLR $\geq 4.5$ or $\triangle N L R \geq 0$ were included in group b; 27 patients with NLR $\geq 4.5$ and $\Delta \mathrm{NLR} \geq 0$ were included in group c.

Median OS was 23.7 (95\% CI: 9.1-38.2) versus 9.3 (95\% CI: 6.0-12.6) versus 4.4 (95\% CI: 2.3-6.5) months for patients in groups a, b, and c, respectively (Figure 1E), and median PFS was 10.8 (95\% CI: 1.0-20.6) versus 4.9 (95\% CI: 3.9-5.8) versus 2.7 (95\% CI: 1.7-3.8) months (both $\mathrm{P}<0.0001$ ) (Figure $1 F$ ). After adjusting for confounding factors, including age, prior chemotherapy, prior target therapy, epidermal growth factor receptor (EGFR) mutation status, antibiotics received within 1 month before, and treatment lines, the multivariate analysis showed that patients with high NLR and $\triangle$ NLR (group c) had a significantly increased risk of death (HR: 10.79, 95\% CI: 4.30-27.10, $\mathrm{P}<0.0001)$ and disease progression (HR: $10.49,95 \%$ CI: 4.39-25.09, $\mathrm{P}<0.0001)$ when compared with patients with
NLR $<4.5$ and $\triangle$ NLR $<0$ (group a) (Table 5). Patients with NLR $\geq 4.5$ or $\triangle N L R \geq 0$ (group b) showed an intermediate risk for both death (HR: 3.12, 95\% CI: 1.35-7.21, P=0.008) and disease progression (HR: 3.45, 95\% CI: 1.62-7.36, $\mathrm{P}=0.001$ ) (Table 5). There was no significant difference for NLR $\geq 4.5$ or $\triangle N L R \geq 0$ in group b (Figure S2). Detailed information of the multivariate analysis is provided in Table S2.

ORR for groups a, b, and c was 53\%, $15 \%$, and $7 \%$, respectively $(\mathrm{P}=0.002)$. DCR for groups $\mathrm{a}, \mathrm{b}$, and $\mathrm{c}$ was $87 \%, 68 \%$, and $41 \%$, respectively $(\mathrm{P}=0.018)$ (Table 2$)$.

\section{Discussion}

In the present study, we demonstrated the efficacy of PD-1 inhibitors in advanced NSCLC patients with poor PS, encountered during real-world practice, with our results suggesting that both high baseline NLR (NLR $\geq 4.5)$ and increased post-treatment NLR $(\triangle N L R \geq 0)$ might be negative prognostic biomarkers of anti-PD-1 treatment in this population. Moreover, the simultaneous elevation of both pretreatment and post-treatment NLR was associated with a significantly increased risk of death and disease progression (Table 5).

Limited data are available on the safety and efficacy of immunotherapy in NSCLC patients with poor PS (16). Only 4 recently published clinical trials (CheckMate 171, CheckMate 153, CheckMate 817, and PePS2) include a small 
A

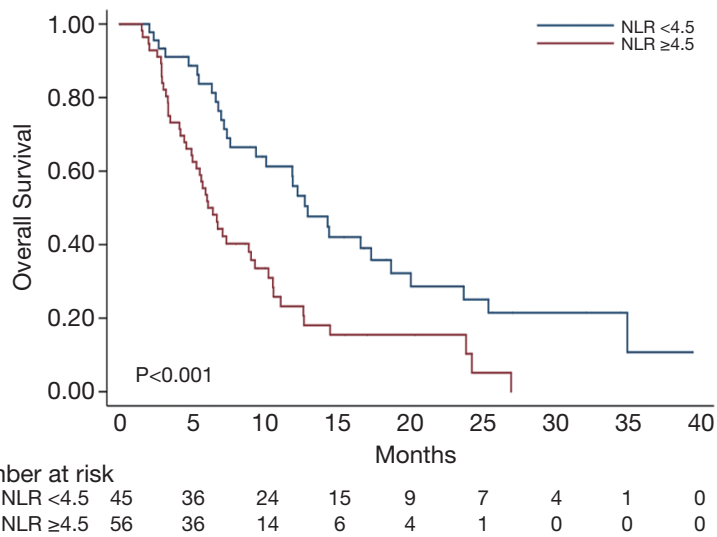

C

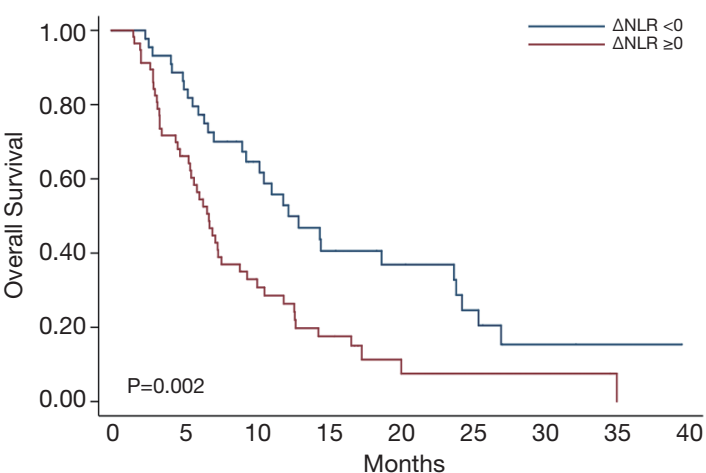

Number at risk

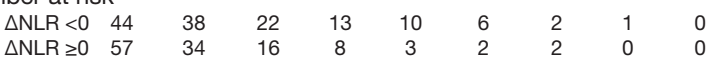

$\mathrm{E}$

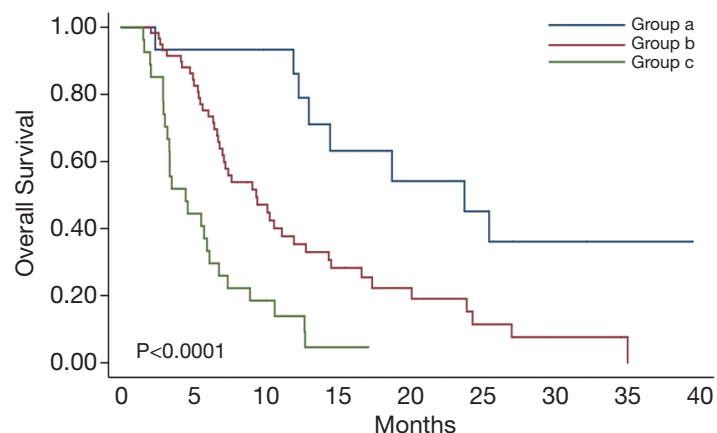

Number at risk

\begin{tabular}{|c|c|c|c|c|c|c|c|}
\hline Group a 15 & 14 & 13 & 8 & 6 & 5 & 2 & 1 \\
\hline Group b 59 & 46 & 20 & 12 & 7 & 3 & 2 & 0 \\
\hline Group c 27 & 12 & 5 & 1 & 0 & 0 & 0 & 0 \\
\hline
\end{tabular}

B

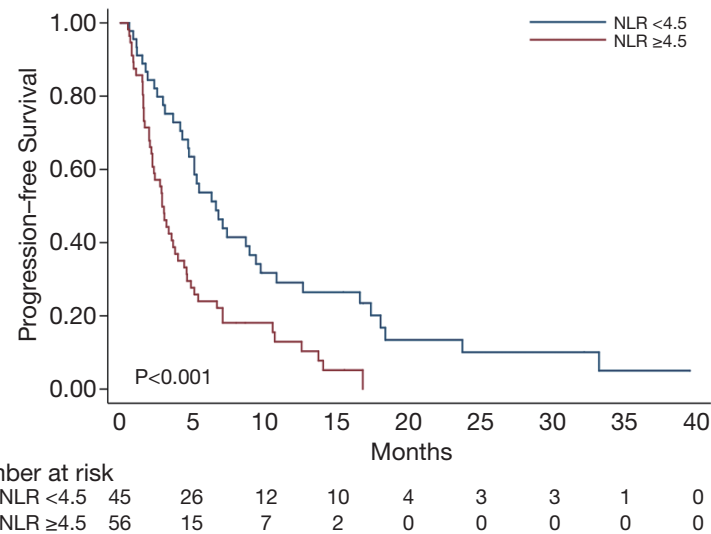

D

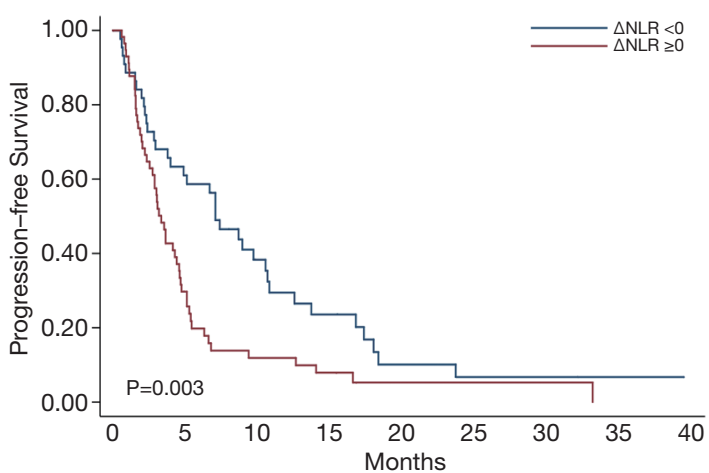

Number at risk

$\begin{array}{llllllllll}\triangle N L R<0 & 44 & 26 & 13 & 8 & 3 & 2 & 2 & 1 & 0 \\ \triangle N L R \geq 0 & 57 & 15 & 6 & 4 & 1 & 1 & 1 & 0 & 0\end{array}$

$\mathrm{F}$

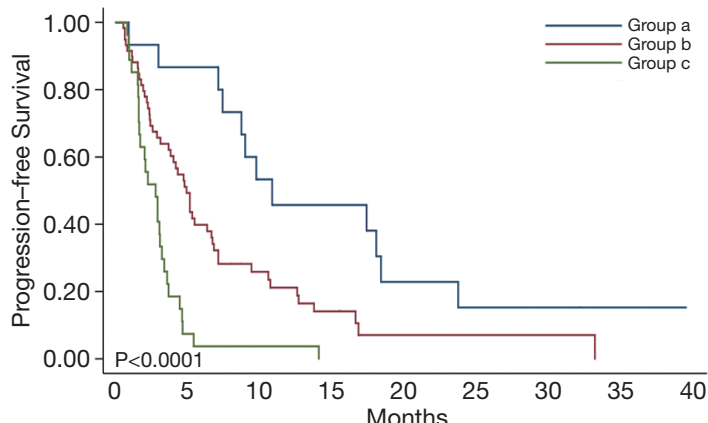

Number at risk

$\begin{array}{ccccccccc}\text { Group a } 15 & 13 & 7 & 6 & 3 & 2 & 2 & 1 & 0 \\ \text { Group b } 59 & 26 & 11 & 6 & 1 & 1 & 1 & 0 & 0\end{array}$

Figure 1 Kaplan-Maier survival curves of advanced NSCLC patients with poor performance status receiving PD-1 inhibitors grouped by each index. (A) OS of patients stratified by pretreatment NLR; (B) PFS of patients stratified by pretreatment NLR; (C) OS of patients stratified by change in NLR ( $\triangle \mathrm{NLR} ; 6$ weeks post-treatment NLR minus baseline NLR); (D) PFS of patients stratified by change in NLR ( $\triangle \mathrm{NLR}$; 6 weeks post-treatment NLR minus baseline NLR); (E) OS of patients stratified by both NLR and $\triangle \mathrm{NLR}$; (F) PFS of patients stratified by both NLR and $\triangle$ NLR. P values are from the log-rank test. NSCLC, non-small cell lung cancer; PD-1, programmed cell death 1; OS, overall survival; NLR, neutrophil-to-lymphocyte ratio; PFS, progression-free survival. 
Table 3 Multivariate analysis of the associations of baseline NLR and survival

\begin{tabular}{|c|c|c|c|c|}
\hline Characteristics & \multicolumn{2}{|c|}{ Overall survival } & \multicolumn{2}{|c|}{ Progression-free survival } \\
\hline Age $\geq 65$ years & $0.77(0.45-1.31)$ & 0.339 & $0.70(0.41-1.19)$ & 0.187 \\
\hline Prior chemotherapy (yes) & $1.47(0.73-2.97)$ & 0.282 & $1.79(0.96-3.32)$ & 0.066 \\
\hline Prior target therapy (yes) & $1.14(0.59-2.21)$ & 0.703 & $1.57(0.86-2.85)$ & 0.140 \\
\hline Antibiotics received within 1 month before & $1.17(0.72-1.91)$ & 0.531 & $1.53(0.96-2.44)$ & 0.074 \\
\hline \multicolumn{5}{|l|}{ Immunotherapy as systemic } \\
\hline 1 & 1 (ref) & & 1 (ref) & \\
\hline 2 & $0.83(0.35-2.0)$ & 0.680 & $0.84(0.39-1.78)$ & 0.643 \\
\hline
\end{tabular}

*, $\mathrm{P}<0.05$ indicates statistical significance. HR, hazards ratio; $\mathrm{Cl}$, confidence interval; EGFR, epidermal growth factor receptor; ref, reference category; NLR, neutrophil-to-lymphocyte ratio.

Table 4 Multivariate analysis of the associations of $\triangle$ NLR and survival

\begin{tabular}{|c|c|c|c|c|}
\hline Characteristics & \multicolumn{2}{|c|}{ Overall survival } & \multicolumn{2}{|c|}{ Progression-free survival } \\
\hline Age $\geq 65$ years & $0.86(0.49-1.49)$ & 0.590 & $0.70(0.40-1.22)$ & 0.203 \\
\hline Prior chemotherapy (yes) & $1.56(0.73-3.36)$ & 0.251 & $2.35(1.21-4.57)$ & $0.012^{*}$ \\
\hline Prior target therapy (yes) & $1.02(0.54-1.91)$ & 0.957 & $1.70(0.96-3.00)$ & 0.070 \\
\hline Antibiotics received within 1 month before & $1.29(0.78-2.12)$ & 0.318 & $1.90(1.18-3.06)$ & $0.009^{*}$ \\
\hline \multicolumn{5}{|l|}{ Immunotherapy as systemic } \\
\hline 1 & 1 (ref) & & 1 (ref) & \\
\hline 2 & $0.96(0.38-2.43)$ & 0.936 & $0.73(0.33-1.62)$ & 0.438 \\
\hline
\end{tabular}

* $\mathrm{P}<0.05$ indicates statistical significance. $\mathrm{HR}$, hazards ratio; $\mathrm{Cl}$, confidence interval; EGFR, epidermal growth factor receptor; ref, reference category; $\triangle N L R, 6$ weeks post-treatment NLR minus baseline NLR.

number of PS 2 NSCLC patients, showing results of ORR, median PFS and OS ranging from $20 \%$ to $27 \%, 3.6$ to 4.4 months, and 4.0 to 9.8 months, respectively (3-5). However, with the exception of PePS2, the populations of the other 3 trials were heterogeneous, consisting of a mix of elderly patients and PS $<2$ patients with comorbidities. These clinical trials also lacked PS 3-4 patients and there is a need for immunotherapy in clinical practice for these patients. Thus, due to the lack of a sufficient number of studies and the inconsistency of the results, the survival benefit in poor PS patients receiving anti-PD-1 therapy remains controversial.

Another challenge is the selection of potential patients likely to benefit from immunotherapy among the poor PS population. In the PePS2 trial, patients with PDL1 $\geq 50 \%$ expression had substantial improvement of ORR (27\% vs. 47\%), PFS (4.4 vs. 12.6 months), and OS 
Table 5 Multivariate analysis of the associations of NLR and $\triangle$ NLR with survival

\begin{tabular}{|c|c|c|c|c|}
\hline Characteristic & \multicolumn{2}{|c|}{ Overall survival } & \multicolumn{2}{|c|}{ Progression-free survival } \\
\hline Group a & 1 (ref) & & 1 (ref) & \\
\hline Group b & $3.12(1.35-7.21)$ & $0.008^{*}$ & $3.45(1.62-7.36)$ & $0.001^{*}$ \\
\hline Group c & $10.79(4.30-27.10)$ & $<0.0001^{*}$ & 10.49 (4.39-25.09) & $<0.0001^{\star}$ \\
\hline
\end{tabular}

*, $\mathrm{P}<0.05$ indicates statistical significance. HR, hazards ratio; $\mathrm{Cl}$, confidence interval; NLR, neutrophil-to-lymphocyte ratio; ref, reference category; $\triangle N L R, 6$ weeks post-treatment NLR minus baseline NLR.

(9.8 vs. 14.6 months) compared with patients with $\mathrm{PD}$ L1 $<1 \%$ expression (4). However, PD-L1 expression was not available for a proportion of the patients due to the absence of tumor tissue, invasiveness of biopsy, and highly expensive detection procedures. Therefore, biomarkers with good quality, as well as high sensitivity and selectivity, dynamically available, non-invasive, and cost-effective are ideal for predicting the efficacy of immunotherapy.

Inflammation has been closely correlated with immunomodulation and the progression of tumors. The systemic inflammatory index, which is derived from routine blood parameters, such as elevated ANC, NLR, derived neutrophil-to-lymphocyte ratio (dNLR), absolute platelet count, platelet-to-lymphocyte ratio (PLR), and lactate dehydrogenase, have been suggested as independent biomarkers of poor outcomes for patients with various cancer types receiving PD-1 inhibitors, including NSCLC (17-22).

In the present study, we investigated the relationship between NLR (or $\triangle N L R$ ) and clinical outcomes of antiPD-1 therapy in advanced NSCLC patients with poor PS from real-world data. In accordance with published clinical trials, our study demonstrated that the ORR, median OS, and PFS were 19\%, 9.0 (95\% CI: 6.3-11.8), and 4.3 (95\% CI: 3.2-5.3) months, respectively. The mechanistic explanation for the prognostic outcomes of the NLR remains unclear. The lymphocyte has been known as the effector cell of antitumor response, and the number of tumor-infiltrating lymphocytes appears to be predictive of response to immune therapies $(23,24)$. Recent studies found that blood neutrophils had the direct link with the number of infiltrated neutrophils, which may have the potential to compromise the antitumor immune response (25). Neutrophils not only target tumor cells but also indirectly affect other cells of the tumor microenvironment, playing an important role in the progression of tumors as well as metastases. This effect is achieved through the secretion and release of various chemokines and cytokines, including transforming growth factor beta, vascular endothelial growth factor, IL-6, and IL-8 (26). Moreover, a latest study demonstrated that there was an association between a higher neutrophil count and decreased CD8+ T cells in NSCLC cells, identifying neutrophilia could inhibit the antitumor immune response by inhibition of the cytotoxic activity of immune cells, particular activated CD8+ T cells (19).

Notably, by analyzing the relationship between various variables and survival, we discovered that both high baseline NLR and increased post-treatment NLR were independently and significantly associated with poor survival outcomes. Furthermore, the simultaneous elevation of both factors were associated with a 10.79-fold increased risk of death and a 10.49-fold increased risk of progression (Table 5). High baseline NLR and increased post-treatment NLR were also significantly associated with reduced ORR and DCR (Table 2). In contrast, among patients who had low baseline NLRs (NLR <4.5), those who had decreased post-treatment NLRs $(\triangle \mathrm{NLR}<0)$ and those who had both would have especially favorable prognoses. To the best of our knowledge, this is the first study to suggest that baseline NLR and post-treatment NLR might be of value as a prognostic biomarker to risk-stratify advanced NSCLC patients with poor PS receiving PD-1 inhibitors.

The present study has several limitations. First, our exploratory evaluation was retrospective and post hoc, which could result in inevitable biases, such as patients being lost to follow-up. Second, the analysis was performed using data from a single institution and an enrollment of a relatively small number of patients relative to the initial recruitment; therefore, the potential for selection bias might exist. Another limitation was the lack of a control cohort not receiving PD-1 inhibitors, which meant that we were unable to establish whether NLR and $\triangle$ NLR were prognostic biomarkers only for anti-PD-1 therapy or general prognostic biomarkers for other treatments. Furthermore, the optimal threshold of NLR and $\triangle N L R$ in advanced NSCLC patients with poor PS, 
and a better prediction model combining NLR and $\triangle$ NLR with other predictive indexes, such as PD-1 expression and $\mathrm{TMB}$, warrant further identification in a large, prospective cohort study.

\section{Conclusions}

To the best of our knowledge, the present study was the first to suggest that high baseline NLR, increased posttreatment NLR, and their combination might serve as an unfavorable prognostic biomarker for outcomes in advanced NSCLC patients with poor PS receiving PD-1 inhibitors, which could support the identification of poor PS patients who are more likely or unlikely to benefit from anti-PD-1 therapy. Further broad-spectrum, prospective studies are warranted for a better understanding of the prognostic value of NLR and $\triangle$ NLR.

\section{Acknowledgments}

The authors appreciate the academic support from AME Lung Cancer Collaborative Group.

Funding: None.

\section{Footnote}

Reporting Checklist: The authors have completed the REMARK reporting checklist. Available at http://dx.doi. org/10.21037/tlcr-21-43.

Data Sharing Statement: Available at http://dx.doi. org/10.21037/tlcr-21-43.

Conflicts of Interest: All authors have completed the ICMJE uniform disclosure form (available at http://dx.doi. org/10.21037/tlcr-21-43). The authors have no conflicts of interest to declare.

Ethical Statement: The authors are accountable for all aspects of the work in ensuring that questions related to the accuracy or integrity of any part of the work are appropriately investigated and resolved. The Institutional Review Board of the Chinese People's Liberation Army General Hospital (Beijing, China) approved the review of the medical records (approval number: S2018-092-01). All procedures performed in this study involving human participants were in accordance with the Declaration of Helsinki (as revised in 2013). Individual consent for this retrospective analysis was waived.

Open Access Statement: This is an Open Access article distributed in accordance with the Creative Commons Attribution-NonCommercial-NoDerivs 4.0 International License (CC BY-NC-ND 4.0), which permits the noncommercial replication and distribution of the article with the strict proviso that no changes or edits are made and the original work is properly cited (including links to both the formal publication through the relevant DOI and the license). See: https://creativecommons.org/licenses/by-nc-nd/4.0/.

\section{References}

1. Su C, Zhou F, Shen J, et al. Treatment of elderly patients or patients who are performance status 2 (PS2) with advanced Non-Small Cell Lung Cancer without epidermal growth factor receptor (EGFR) mutations and anaplastic lymphoma kinase (ALK) translocations - Still a daily challenge. Eur J Cancer 2017;83:266-78.

2. Lilenbaum RC, Cashy J, Hensing TA, et al. Prevalence of poor performance status in lung cancer patients: implications for research. J Thorac Oncol 2008;3:125-9.

3. Felip E, Ardizzoni A, Ciuleanu T, et al. CheckMate 171: A phase 2 trial of nivolumab in patients with previously treated advanced squamous non-small cell lung cancer, including ECOG PS 2 and elderly populations. Eur J Cancer 2020;127:160-72.

4. Middleton G, Brock K, Savage J, et al. Pembrolizumab in patients with non-small-cell lung cancer of performance status 2 (PePS2): a single arm, phase 2 trial. Lancet Respir Med 2020;8:895-904.

5. Spigel DR, McCleod M, Jotte RM, et al. Safety, Efficacy, and Patient-Reported Health-Related Quality of Life and Symptom Burden with Nivolumab in Patients with Advanced Non-Small Cell Lung Cancer, Including Patients Aged 70 Years or Older or with Poor Performance Status (CheckMate 153). J Thorac Oncol 2019;14:1628-39.

6. Barlesi F, Audigier-Valette C, Felip E, et al. OA04.02 CheckMate 817: First-Line Nivolumab + Ipilimumab in Patients with ECOG PS 2 and Other Special Populations with Advanced NSCLC. J Thorac Oncol 2019;14:S214-5.

7. Ready N, Hellmann MD, Awad MM, et al. First-Line Nivolumab Plus Ipilimumab in Advanced Non-SmallCell Lung Cancer (CheckMate 568): Outcomes by Programmed Death Ligand 1 and Tumor Mutational Burden as Biomarkers. J Clin Oncol 2019;37:992-1000.

8. Bagley SJ, Kothari S, Aggarwal C, et al. Pretreatment 
neutrophil-to-lymphocyte ratio as a marker of outcomes in nivolumab-treated patients with advanced non-small-cell lung cancer. Lung Cancer 2017;106:1-7.

9. Russo A, Russano M, Franchina T, et al. Neutrophil-toLymphocyte Ratio (NLR), Platelet-to-Lymphocyte Ratio (PLR), and Outcomes with Nivolumab in Pretreated NonSmall Cell Lung Cancer (NSCLC): A Large Retrospective Multicenter Study. Adv Ther 2020;37:1145-55.

10. Cannon NA, Meyer J, Iyengar P, et al. Neutrophillymphocyte and platelet-lymphocyte ratios as prognostic factors after stereotactic radiation therapy for early-stage non-small-cell lung cancer. J Thorac Oncol 2015;10:280-5.

11. Liu D, Jin J, Zhang L, et al. The Neutrophil to Lymphocyte Ratio May Predict Benefit from Chemotherapy in Lung Cancer. Cell Physiol Biochem 2018;46:1595-605.

12. Mandaliya H, Jones M, Oldmeadow C, et al. Prognostic biomarkers in stage IV non-small cell lung cancer (NSCLC): neutrophil to lymphocyte ratio (NLR), lymphocyte to monocyte ratio (LMR), platelet to lymphocyte ratio (PLR) and advanced lung cancer inflammation index (ALI). Transl Lung Cancer Res 2019;8:886-94.

13. Guo M, Li W, Li B, et al. Prognostic value of delta inflammatory biomarker-based nomograms in patients with inoperable locally advanced NSCLC. Int Immunopharmacol 2019;72:395-401.

14. Dusselier M, Deluche E, Delacourt N, et al. Neutrophilto-lymphocyte ratio evolution is an independent predictor of early progression of second-line nivolumab-treated patients with advanced non-small-cell lung cancers. PLoS One 2019;14:e0219060.

15. Petrova MP, Eneva MI, Arabadjiev JI, et al. Neutrophil to lymphocyte ratio as a potential predictive marker for treatment with pembrolizumab as a second line treatment in patients with non-small cell lung cancer. Biosci Trends 2020;14:48-55.

16. Gounant V, Lavolé A, Quoix E. Ongoing challenges of using immunotherapy in special populations: Poor performance status patients, elderly patients, and people

Cite this article as: Chen S, Li R, Zhang Z, Huang Z, Cui P, Jia W, Zhang S, Tao H, Wang L, Li X, Wang J, Ma J, Liu Z, Huang D, Zheng X, Saito Y, Ichiki Y, Hu Y. Prognostic value of baseline and change in neutrophil-to-lymphocyte ratio for survival in advanced non-small cell lung cancer patients with poor performance status receiving PD-1 inhibitors. Transl Lung Cancer Res 2021;10(3):1397-1407. doi: 10.21037/tlcr-21-43 living with HIV. Lung Cancer 2020;145:71-5.

17. Ferrucci PF, Gandini S, Battaglia A, et al. Baseline neutrophil-to-lymphocyte ratio is associated with outcome of ipilimumab-treated metastatic melanoma patients. Br J Cancer 2015;112:1904-10.

18. Mezquita L, Auclin E, Ferrara R, et al. Association of the Lung Immune Prognostic Index With Immune Checkpoint Inhibitor Outcomes in Patients With Advanced Non-Small Cell Lung Cancer. JAMA Oncol 2018;4:351-7.

19. Kargl J, Busch SE, Yang GH, et al. Neutrophils dominate the immune cell composition in non-small cell lung cancer. Nat Commun 2017;8:14381.

20. Diem S, Kasenda B, Spain L, et al. Serum lactate dehydrogenase as an early marker for outcome in patients treated with anti-PD-1 therapy in metastatic melanoma. Br J Cancer 2016;114:256-61.

21. Jiang $M$, Peng $W, P u X$, et al. Peripheral Blood Biomarkers Associated With Outcome in Non-small Cell Lung Cancer Patients Treated With Nivolumab and Durvalumab Monotherapy. Front Oncol 2020;10:913.

22. Ichiki Y, Taira A, Chikaishi Y, et al. Prognostic factors of advanced or postoperative recurrent non-small cell lung cancer targeted with immune check point inhibitors. J Thorac Dis 2019;11:1117-23.

23. Trujillo JA, Sweis RF, Bao R, et al. T Cell-Inflamed versus Non-T Cell-Inflamed Tumors: A Conceptual Framework for Cancer Immunotherapy Drug Development and Combination Therapy Selection. Cancer Immunol Res 2018;6:990-1000.

24. Pagès F, Mlecnik B, Marliot F, et al. International validation of the consensus Immunoscore for the classification of colon cancer: a prognostic and accuracy study. Lancet 2018;391:2128-39.

25. Moses K, Brandau S. Human neutrophils: Their role in cancer and relation to myeloid-derived suppressor cells. Semin Immunol 2016;28:187-96.

26. Treffers LW, Hiemstra IH, Kuijpers TW, et al. Neutrophils in cancer. Immunol Rev 2016;273:312-28. 


\section{Supplementary}

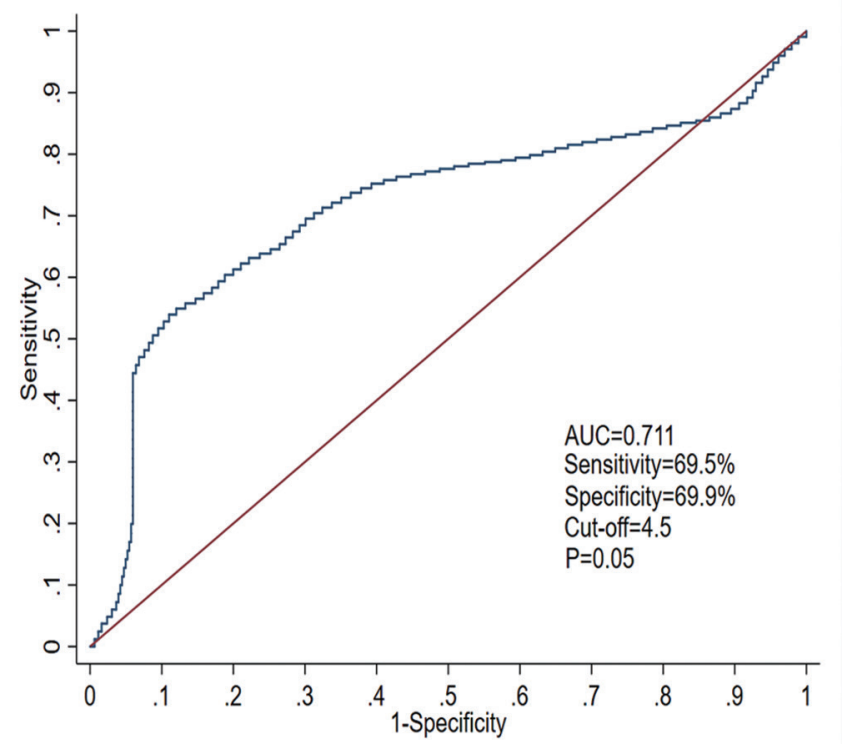

Figure S1 Time-dependent receiver-operating curve of baseline neutrophil-to-lymphocyte ratio for overall survival. AUC, area under the curve.

A

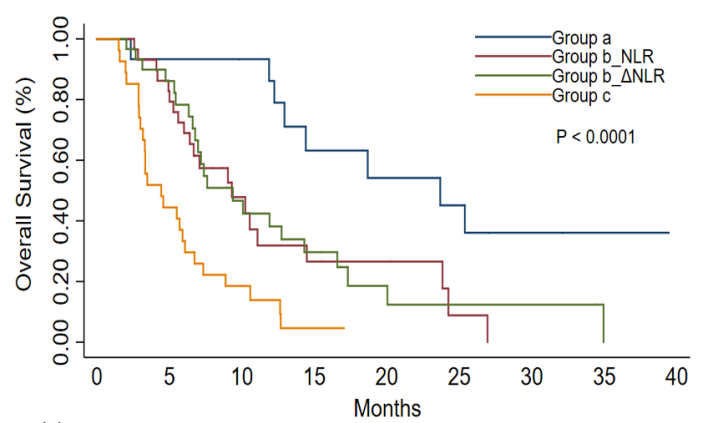

B

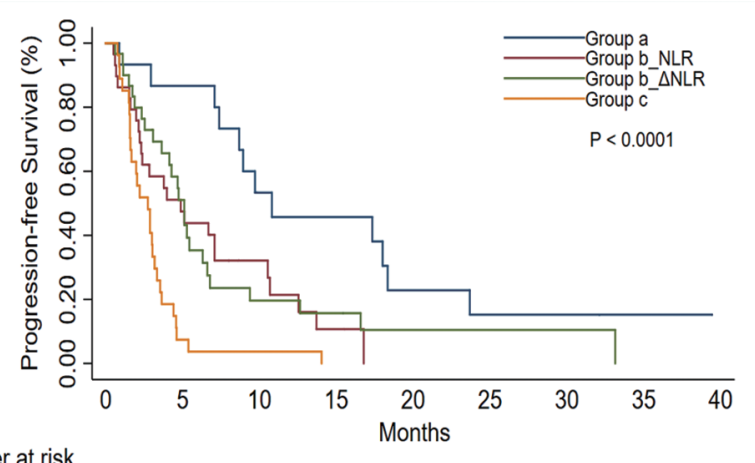

Number at risk

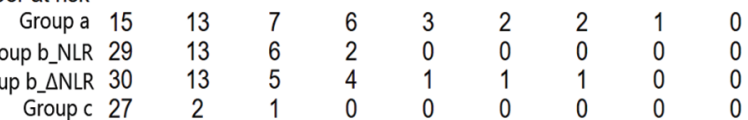

Figure S2 Kaplan-Meier curves of overall survival and progression-free survival for patients stratified by baseline neutrophil-to-lymphocyte ratio (NLR) and 6 weeks post-treatment NLR minus baseline NLR ( $\triangle N L R$ ). (A) Overall survival; (B) progression-free survival. 
Table S1 Univariate analyses of factors associated with overall survival and progression-free survival

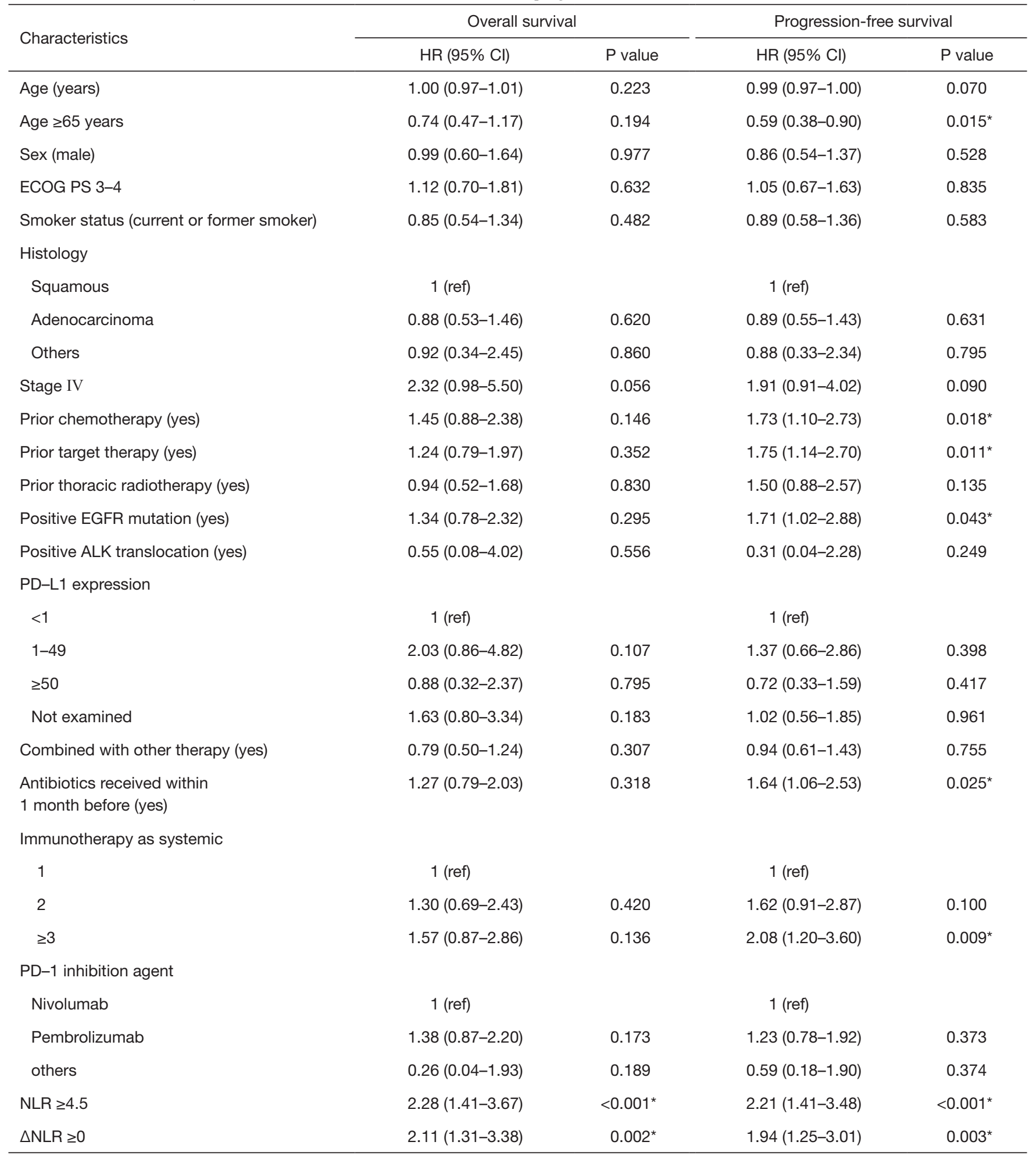

*, $\mathrm{P}<0.05$ indicates statistical significance. ALK, anaplastic lymphoma kinase; Cl, confidence interval; CNS, central nervous system; ECOG PS, Eastern Cooperative Oncology Group Performance Status; EGFR, epidermal growth factor receptor; HR, hazards ratio; NLR, neutrophil-to-lymphocyte ratio; PD-1, programmed cell death 1; ref, reference category; $\triangle N L R, 6$ weeks post-treatment NLR minus baseline NLR. 
Table S2 Multivariate analysis of the associations of NLR and $\triangle$ NLR with survival

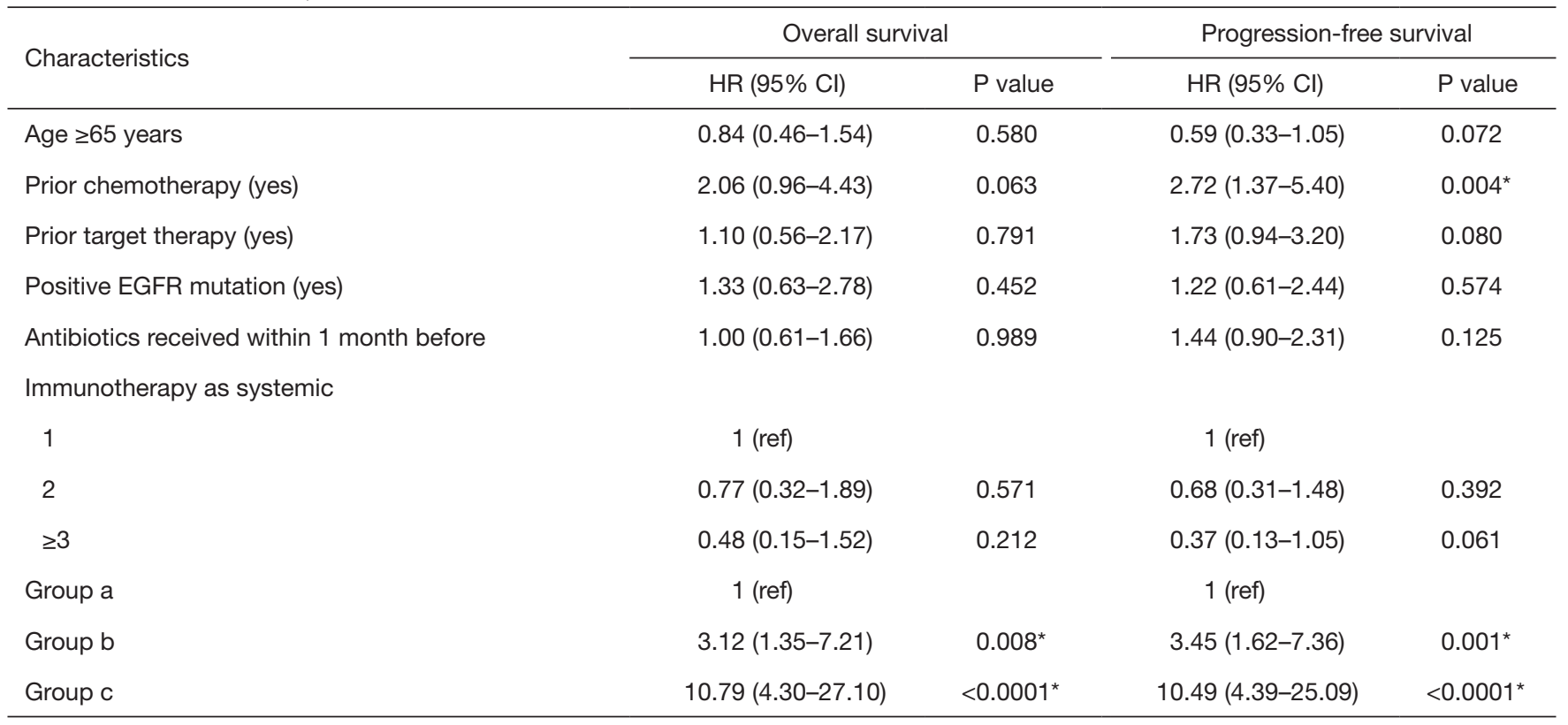

* $\mathrm{P}<0.05$ indicates statistical significance. $\mathrm{Cl}$, confidence interval; EGFR, epidermal growth factor receptor; HR, hazards ratio; NLR, neutrophil-to-lymphocyte ratio; ref, reference category; $\triangle N L R, 6$ weeks post-treatment NLR minus baseline NLR. 